http://jmscr.igmpublication.org/home/

ISSN (e)-2347-176x ISSN (p) 2455-0450

crossref DOI: https://dx.doi.org/10.18535/jmscr/v9i11.26

Journal Of Medical Science And Clinical Research

\title{
A Rare Phenomenon of Thrombocytopenia in Oleander Seed Poisoning
}

\author{
Authors \\ Dr Kaverikannan MD ${ }^{1}$, Dr Reeba Thannickal Mathews ${ }^{2}$ \\ ${ }^{1}$ Associate professor, Department of General Medicine, Kanyakumari Government Medical College and \\ Hospital, Asaripallam, Tamil Nadu \\ ${ }^{2}$ Post Graduate, Department of General Medicine, Kanyakumari Government Medical College and Hospital, \\ Asaripallam, Tamil Nadu

\begin{abstract}
This is a case series of 5 patients who were admitted in a tertiary care centre, in South India, over the period of two years with oleander seed poisoning, who during the course in the hospital developed significant thrombocytopenia. In all the cases, thrombocytopenia occurred suddenly, within days of presentation and was rapidly deteriorating in nature. Hence enough monitoring is required from the part of treating physician to prevent severe bleeding manifestation. Even though thrombocytopenia is very rare, more studies have to be conducted in this matter.
\end{abstract}

\section{Introduction}

Oleander, Nerium oleander, is an ornamental plant, seen in almost all over India. Poisoning with common oleander (Nerium oleander) is more frequent than yellow oleander (Thevetia peruviana). It is well known that all parts of the Oleander plant are poisonous, which includes flower, leaf and stem. The kernel is almost seven times morepoisonous as compared to leaves, stems, flowers or fruit pulp. Poisonous ingredients include: Digitoxigenin, Neriin, Oleandrin, Oleondroside. Oleandrin and oleandrigenin are cardiac glycosides, and are toxic to human body. Oleander seed poisoning ranks the first place in plant poisoning in South India and second in the poisoning cases after organophosphorus poisoning (1)

Just like the digoxin, which has very narrow therapeutic index, mild variations in the serum toxin levels will significantly affect toxic manifestations. Since absorption appears to be slow and erratic, volume of distribution has not been studied, fatal dose calculation is variable. Starting with the gastrointestinal effects which includes nausea and vomiting, abdominal pain, diarrhoea. Cardiac reactions consist of irregularities in heart rate, The effect on the central nervous system include symptoms such as drowsiness, tremors, seizures.

Even though cases of thrombocytopenia is described in patient with acute digitoxin toxicity ${ }^{(2)}$ and patients on routine digitoxin, mechanism and predictability of it is not explored much. Studies of thrombocytopenia in oleander seed poisoning is also not conducted much, except for the occasionally reported cases ${ }^{(3)}$ 


\section{Case Reports}

Among the cases of oleander seed poisoning admitted in our hospital in the year of 2019-2020, 5 cases of significant thrombocytopenia had been noted. Patients on antiplatelets, heparin for any reason, chronic liver disease, patients on any bone marrow suppressive drugs, patients with anemia and leukopenia and patients with splenomegaly were all excluded from the analysis.

All the 5 cases were brought to ER within 6 hrs of poison ingestion. Within the $10 \mathrm{~min}$ of presentation, all were given thorough stomach wash and activated charcoal as per the standard protocol for the oleander seed poisoning. All the patients were kept in intensive care unit, under continuous cardiac monitoring. Base line investigations were daily monitored.
Out of the five patients in whom significant thrombocytopenia noted, all of them had platelet count below one lakh/ $\mu \mathrm{L}$. In all the patients, dip in platelet count noticed within first 5 days, and count returned to normal limits within first week itself. Two patients developed bleeding manifestations with platelet count less than $20000 / \mu \mathrm{L}$, and they required platelet transfusion. None of the patients were given heparin, no pacemaker insertion done, Fab not given. Evaluation done for thrombocytopenia, serology tests for common viral infections implicated for thrombocytopenia done which were all negative in all patients. Examination of peripheral blood smears showed normal red blood cells and leucocytes, and thrombocytopenia.

\begin{tabular}{|c|c|c|c|c|c|c|c|c|}
\hline $\begin{array}{l}\text { Case } \\
\text { No }\end{array}$ & Age & Sex & $\begin{array}{l}\text { Time } \\
\text { taken to } \\
\text { reach } \\
\text { hospital }\end{array}$ & $\begin{array}{c}\text { Treatment } \\
\text { initiated } \\
\text { within }\end{array}$ & $\begin{array}{l}\text { No of } \\
\text { seeds } \\
\text { taken }\end{array}$ & $\begin{array}{c}\text { Pulse rate/ } \\
\text { blood } \\
\text { pressure }\end{array}$ & $\begin{array}{c}\text { ECG } \\
\text { (on admission) }\end{array}$ & $\begin{array}{c}\text { ECG } \\
\text { (after } 24 \mathrm{hrs} \text { ) }\end{array}$ \\
\hline 1. & 23 & $\mathrm{~m}$ & $2 \mathrm{hrs}$ & $10 \mathrm{~min}$ & 7 & $\begin{array}{l}56 / \mathrm{min} \\
132 / 86 \\
\mathrm{mmHg}\end{array}$ & Sinus bradycardia & $\begin{array}{l}\text { Type } 1 \text { second degree AV } \\
\text { block,reverse tick sign }\end{array}$ \\
\hline 2. & 26 & $\mathrm{f}$ & 3.5 & $10 \mathrm{~min}$ & 2 & $\begin{array}{l}48 / \mathrm{min} \\
112 / 70 \\
\mathrm{mmHg}\end{array}$ & $\begin{array}{c}\text { Sinus } \\
\text { bradycardia }\end{array}$ & Sinus bradycardia \\
\hline 3. & 34 & $f$ & $1.5 \mathrm{hrs}$ & $10 \mathrm{~min}$ & 4 & $\begin{array}{l}74 / \mathrm{min} \\
100 / 68 \\
\mathrm{mmHg}\end{array}$ & Sinus rhythm & Sinus bradycardia \\
\hline 4. & 28 & $\mathrm{~m}$ & $6 \mathrm{hrs}$ & $10 \mathrm{~min}$ & 3 & $\begin{array}{l}52 / \mathrm{min} \\
130 / 88 \\
\mathrm{mmHg} \\
\end{array}$ & Sinus rhythm & Sinus bradycardia \\
\hline 5. & 47 & $\mathrm{f}$ & $2 \mathrm{hrs}$ & $10 \mathrm{~min}$ & 6 & $\begin{array}{l}\text { 48/min } \\
110 / 74 \\
\mathrm{mmHg}\end{array}$ & $\begin{array}{l}\text { First degree AV } \\
\text { block }\end{array}$ & First degree AV block \\
\hline
\end{tabular}

\begin{tabular}{|c|c|c|c|c|c|}
\hline $\begin{array}{l}\text { Case } \\
\text { No }\end{array}$ & $\begin{array}{l}\text { Platelet } \\
\text { at the presentation }\end{array}$ & $\begin{array}{l}\text { Lowest Platelet } \\
\text { Count noted (per } \\
\mu \mathrm{L} \text { of blood) }\end{array}$ & $\begin{array}{c}\text { Platelet count returned } \\
\text { to values above } 1.5 \mathrm{~L} \\
\text { on }\end{array}$ & Transfusion needed & Bleeding manifestations \\
\hline 1. & $3.75 \mathrm{~L}$ & $\begin{array}{c}7000 \\
\left(4^{\text {th }} \text { day }\right)\end{array}$ & 8th day & 4 units & $\begin{array}{c}\text { Yes, } \\
\text { Gum bleed }\end{array}$ \\
\hline 2. & $2.82 \mathrm{~L}$ & $\begin{array}{c}56000 \\
\left(5^{\text {th }} \text { day }\right)\end{array}$ & $7^{\text {th }}$ day & nil & nil \\
\hline 3. & $1.91 \mathrm{~L}$ & $\begin{array}{c}96000 \\
\left(3^{\text {rd }} \text { day }\right)\end{array}$ & $5^{\text {th }}$ day & nil & nil \\
\hline 4. & $2.46 \mathrm{~L}$ & $\begin{array}{c}13000 \\
\left(3^{\text {rd }} \text { day) }\right.\end{array}$ & $6^{\text {th }}$ day & 4 units & $\begin{array}{l}\text { Yes, ecchymosis at the } \\
\text { injection site }\end{array}$ \\
\hline 5. & $3.80 \mathrm{~L}$ & $\begin{array}{c}92000 \\
\left(4^{\text {th }} \text { day }\right)\end{array}$ & $6^{\text {th }}$ day & nil & nil \\
\hline
\end{tabular}


Serial Platelet Counts (per $\mu \mathrm{L}$ of blood)

\begin{tabular}{|l|c|c|c|c|c|c|c|c|c|c|}
\hline $\begin{array}{l}\text { Case } \\
\text { no }\end{array}$ & $\begin{array}{c}\text { Platelet } \\
\text { on day 1 }\end{array}$ & $\begin{array}{c}\text { Platelet } \\
\text { on day } \\
2\end{array}$ & $\begin{array}{c}\text { Platelet } \\
\text { on day } \\
3\end{array}$ & $\begin{array}{c}\text { Platelet } \\
\text { on day 4 }\end{array}$ & $\begin{array}{c}\text { Platelet } \\
\text { on day } \\
5\end{array}$ & $\begin{array}{c}\text { Platelet } \\
\text { on day } \\
6\end{array}$ & $\begin{array}{c}\text { Platelet } \\
\text { on day } \\
7\end{array}$ & $\begin{array}{c}\text { Platelet } \\
\text { on day } \\
8\end{array}$ & $\begin{array}{c}\text { Platelet } \\
\text { on day } \\
9\end{array}$ & $\begin{array}{c}\text { Platelet } \\
\text { on day 10 }\end{array}$ \\
\hline & $3.75 \mathrm{~L}$ & $1.62 \mathrm{~L}$ & 59000 & $\mathbf{7 0 0 0}$ & 44000 & 87000 & $1.35 \mathrm{~L}$ & $2.04 \mathrm{~L}$ & $2.47 \mathrm{~L}$ & $2.53 \mathrm{~L}$ \\
\hline 2 & $2.82 \mathrm{~L}$ & $3.04 \mathrm{~L}$ & $2.35 \mathrm{~L}$ & 79000 & $\mathbf{5 6 0 0 0}$ & $1.24 \mathrm{~L}$ & $2.64 \mathrm{~L}$ & $2.48 \mathrm{~L}$ & $2.57 \mathrm{~L}$ & $2.78 \mathrm{~L}$ \\
\hline 3 & $1.91 \mathrm{~L}$ & $1.23 \mathrm{~L}$ & $\mathbf{9 6 0 0 0}$ & 98000 & $1.67 \mathrm{~L}$ & $2.38 \mathrm{~L}$ & $2.52 \mathrm{~L}$ & $2.76 \mathrm{~L}$ & $2.91 \mathrm{~L}$ & $2.82 \mathrm{~L}$ \\
\hline 4 & $2.46 \mathrm{~L}$ & 79000 & $\mathbf{1 3 0 0 0}$ & 48000 & 99000 & $1.59 \mathrm{~L}$ & $1.93 \mathrm{~L}$ & $2.36 \mathrm{~L}$ & $2.47 \mathrm{~L}$ & $2.58 \mathrm{~L}$ \\
\hline 5 & $3.80 \mathrm{~L}$ & $2.81 \mathrm{~L}$ & $1.44 \mathrm{~L}$ & $\mathbf{9 2 0 0 0}$ & 98000 & $2.23 \mathrm{~L}$ & $2.86 \mathrm{~L}$ & $2.97 \mathrm{~L}$ & $2.78 \mathrm{~L}$ & $2.79 \mathrm{~L}$ \\
\hline
\end{tabular}

\section{Conclusion}

Oleander poison ranks as the second most common poisoning in south India. Cardiac arrhythmias, heart blocks due to the cardiac glycosides are the most dreaded complication. Thrombocytopenia as a complication of oleander poisoning is not well studied, eventhough it was described as an unpredictable, rare, idiosyncratic complication in digoxin toxicity.

Thrombocytopenia can be seen in both acute overdosing of digoxin and also in chronic toxicity. The idiosyncratic nature of this complication makes it difficult to predict it. Studies for the association of dose of oleander poison intake, time gap in initiation of treatment and severity of thrombocytopenia are not well established due to the lack of appropriate studies conducted in this regard. Taking into consideration the huge caseload of oleander seed poisoning, it is not prudent to disregard this as a rare phenomenon, as Fab, the only known antidote for this poisoning is not readily available and affordable in our country.

\section{Bibliography}

1. Gopalakrishnan, S.K., Kandasamy,Issac etal.Oleander toxicity- the clinical spectrum and mortality predictors: An observational study. Internet Journal of Medical Update- EJOURNAL, 12(1),4. Doi:10.4314/ijmu.v12i1.2
2. Pirovino M, Ohnhaus EE,von Felton A.Digoxin- associated thrombocytopenia. Eur J Clin Pharmacol.1981 Feb:19(3):2057.doi:

10.1007/BF00561950.PMID:7215418

3. Bataille C, Capaldo L, Courtois A, Seguy B, Lotiron C, Labadie M.Toxic thrombocytopenia during Nerium oleander poisoning.Clin Toxicol (Phila).2018 Nov;56(11):1170-

1171.doi:10.1080/15563650.2018.147358

2.Epub2018 May 15.PMID: 29764236. 